\title{
Future Changes in the Global Frequency of Tropical Cyclone Seeds
}

\author{
Masato Sugi ${ }^{1}$, Yohei Yamada ${ }^{2}$, Kohei Yoshida ${ }^{1}$, Ryo Mizuta ${ }^{1}$, \\ Masuo Nakano ${ }^{2}$, Chihiro Kodama ${ }^{2}$, and Masaki Satoh ${ }^{3}$ \\ ${ }^{1}$ Meteorological Research Institute, Tsukuba, Japan \\ ${ }^{2} J a p a n$ Agency for Marine-Earth Science and Technology, Yokohama, Japan \\ ${ }^{3}$ Atmosphere and Ocean Research Institute, University of Tokyo, Kashiwa, Japan
}

\begin{abstract}
In relation to projections of tropical cyclone (TC) frequency in a future warmer climate, there is a debate on whether the global frequency of TC seeds (weak pre-storm vortices) will increase or not. We examined changes in the frequency of TC seeds by occurrence frequency analysis (OFA) of vortex intensity (vorticity or maximum wind speed). We directly counted the number of vortices with various intensities in high resolution global atmospheric model simulations for present and future climates. By using the OFA we showed a clear reduction of the occurrence frequency of TC seeds and relatively weak (category 2 or weaker) TCs in a future warmer climate, with an increase in the frequency of the most intense (category 5) TCs. The results suggest that the OFA is a useful method to estimate the future changes in TC frequency distribution ranging from TC seeds to the most intense TCs.

(Citation: Sugi, M., Y. Yamada, K. Yoshida, R. Mizuta, M. Nakano, C. Kodama, and M. Satoh, 2020: Future changes in the global frequency of tropical cyclone seeds. SOLA, 16, 70-74, doi:10.2151/sola.2020-012.)
\end{abstract}

\section{Introduction}

In a recent review paper on tropical cyclone (TC) and climate change assessment by Knutson et al. (2020), authors' opinion was divided on the confidence level of the projected decrease in global TC frequency in a future warmer climate. Although a majority of the models projected a decrease, two studies projected a clear increase in the global TC frequency. Emanuel (2013) showed a clear increasing trend in global TC frequency during 150 years from mid-20th century to the end of 21 st century, in contrast to a clear decreasing trend during 228 years from late 19th century to the end of 21 st century in a high resolution $(60 \mathrm{~km})$ global atmospheric model shown by Sugi and Yoshimura (2012). Bhatia et al. (2018) also showed a clear increase in global TC frequency in late 21 st century by using a high resolution $(25 \mathrm{~km})$ global atmosphere ocean coupled model.

In relation to these studies, there is a debate on whether the frequency of TC seeds (weak pre-storm vortices) will increase or not in the future warm climate. Emanuel (2013) assumes that the number of TC seeds does not change as climate warms, and argues that the TC frequency increases because the probability of TC genesis from the seeds (survival rate) increases in a warmer climate. Vecchi et al. (2019) examined the reason for the changes in TC frequency in the experiments by Bhatia et al. (2018). They argue that the global TC frequency will increase because the number of TC seeds will increase in a future warmer climate, although the survival rate of TC seeds will decrease. They estimated the TC seeds frequency and survival rate by using a statistical method. In this study, we examined the changes in TC seeds by directly counting the number of TC seeds in the simulations of two high resolution global atmosphere models by using an occurrence frequency analysis (OFA) method.

Corresponding author: Masato Sugi, Meteorological Research Institute, 1-1 Nagamine Tsukuba, Ibaraki 305-0052, Japan, E-mail: msugi@mri-jma. go.jp.

CThe Author(s) 2020. This is an open access article published by the Meteorological Society of Japan under a Creative Commons Attribution 4.0 International (CC BY 4.0) license (http://creativecommons.org/license/by/4.0).

\section{Data and method}

\subsection{Data}

The simulation data used in this study are climate projection experiments using two high resolution global atmospheric models: $20 \mathrm{~km}$ resolution MRI-AGCM3.2 (Mizuta et al. 2012) and 14km resolution NICAM (Satoh et al. 2008; Satoh et al. 2014). The MRI-AGCM experiment consists of a 30 year present climate simulation for 1979-2008 and a 25 year future climate simulation for 2075-2099 (Mizuta et al. 2012; Ogata et al. 2016), while the NICAM experiment consists of a 30 year present climate simulation for 1979-2008 and a 30 year future climate simulation for 2075-2104 (Satoh et al. 2015; Kodama et al. 2015; Yamada et al. 2017). Note that the MRI-AGCM experiment used the future SST data based on the ensemble mean of the Coupled Model Intercomparison Project Phase 5 (CMIP5; Taylor et al. 2012) models with the RCP 8.5 scenario $\left(3.7^{\circ} \mathrm{C}\right.$ global mean surface temperature increase from present), while the NICAM experiment used the future SST data based on the ensemble mean of the Coupled Model Intercomparison Project Phase 3 (CMIP3; Meehl et al. 2007) models with the A1B scenario $\left(2.65^{\circ} \mathrm{C}\right.$ global mean surface temperature increase from present). Radiative forcing prescribed in MRI-AGCM and NICAM experiments are based on A1B scenario and RCP8.5 scenario, respectively. Also note that we used the first 25 years of the simulation data for our analysis.

The observation data we used in this study is the $1.25^{\circ}$ longitude-latitude grid reanalysis data JRA-55 (Kobayashi et al. 2015). For the observed TC tracking data, we used best-track datasets compiled by the International Best Track Archive for Climate Stewardship (IBTrACS; Knapp et al. 2010).

\subsection{Occurrence frequency analysis}

We calculated occurrence frequency of vortex intensity (vorticity at $850 \mathrm{hPa}$ or maximum surface wind speed) in 6 hourly simulation data. Figure 1 shows a snapshot of vorticity at $850 \mathrm{hPa}$ and $500 \mathrm{hPa}$ together with cloud water content at $500 \mathrm{hPa}$ over the tropical North Pacific at 0000 UTC August 2004. Supplement Figure $\mathrm{S} 1$ is a one month animation of the same figure. We can see many westward propagating weak vortices, only a few of which develop into intense vortices with TC strength. In this study, we regard a tall weak $850 \mathrm{hPa}$ vortex with warm core structure as a TC seed. If the ratio of the vorticity at $500 \mathrm{hPa}$ to the vorticity at $850 \mathrm{hPa}$ is between 0.5 and 1.0 , then the vortex is regarded as a TC seed or a TC. In this study we define a TC seed as the $850 \mathrm{hPa}$ vortex with surface maximum wind between $10 \mathrm{~m} \mathrm{~s}^{-1}$ and $17 \mathrm{~m} \mathrm{~s}^{-1}$, and a TC as $850 \mathrm{hPa}$ vortex with surface maximum wind larger than $17 \mathrm{~m} \mathrm{~s}^{-1}$. We counted number of such $850 \mathrm{hPa}$ vortices with various intensities in each 6 hourly snapshot of the simulated data or the reanalysis data on $1.25^{\circ}$ latitude-longitude grid. To calculate the occurrence frequency of the maximum surface wind speed associated with a vortex, we used the original model resolution (20 km grid for MRI-AGCM and $14 \mathrm{~km}$ grid for NICAM) surface wind data within $200 \mathrm{~km}$ from the vortex center. One year total occurrence frequency thus calculated from 6 hourly data is equal to 4 times "Vortex days" or "TC days" per year. 


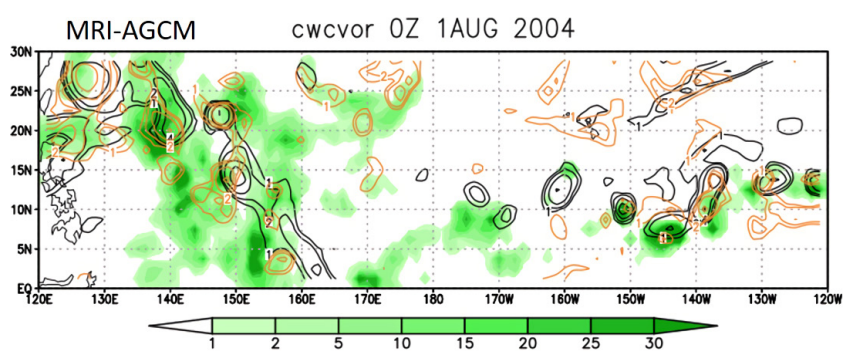

tribution of the simulations for present climate and future warmer climate for MRI-AGCM and NICAM, respectively. The both models show a clear reduction of the occurrence frequency in the future warmer climate with larger reduction in the weaker vorticity. The reduction of the frequency is also clearly shown in the exceedance frequency (total frequency exceeding a certain value) distributions (Figs. 2e and 2f).

In order to see the occurrence frequency of TC seeds (defined as $850 \mathrm{hPa}$ vortices with surface maximum wind between $10 \mathrm{~m}^{-1}$ and $17 \mathrm{~m}^{-1}$ ), we next examined the occurrence frequency of surface maximum wind speed associated with the vortices at $850 \mathrm{hPa}$. Figure 3a shows occurrence frequency distributions for MRI-AGCM and NICAM present climate simulations. Note that the occurrence frequency distribution for JRA-55 is not shown, because the high resolution surface wind data is not available for JRA-55. For the weaker maximum wind speed less than $20 \mathrm{~m} \mathrm{~s}^{-1}$ the frequency of NICAM is considerably larger than that of MRIAGCM, probably due to the higher resolution of NICAM with too many vortices associated with too intense grid scale convections. Figures $3 b$ and $3 c$ show the occurrence frequency distribution of the surface wind speed in the simulations for present climate and future warmer climate for MRI-AGCM and NICAM, respectively. Both models show a clear reduction of the occurrence frequency in the future warmer climate with larger reduction in the weaker wind speeds. Table 1 shows the occurrence frequency (TC days per year) of each TC category. Both models show a clear reduction of TC seeds frequency in the future warmer climate. The rate of reduction of TC seeds in NICAM is considerably smaller than that in MRI-AGCM, but both of them are highly statistically significant. We can define survival rate of TC seeds as the ratio of TS frequency to TC seeds frequency. In the MRI-AGCM present and future climate simulations, the survival rate is $18 \%$ and $17 \%$, respectively, while in the NICAM simulations, the survival rate is $31 \%$ and $27 \%$, respectively. In both models, the future survival rate is a little less than the present survival rate.

\subsection{TC occurrence frequency}

Next, we examined the occurrence frequency of TCs. Figure 4 shows occurrence frequency distributions of the maximum surface wind speed as in Fig. 3 but over the wind speed ranging from $15 \mathrm{~m} \mathrm{~s}^{-1}$ to $80 \mathrm{~m} \mathrm{~s}^{-1}$ with $5 \mathrm{~m} \mathrm{~s}^{-1}$ bin size. Note that the frequencies are different in Fig. 3 and Fig. 4 because the bin size
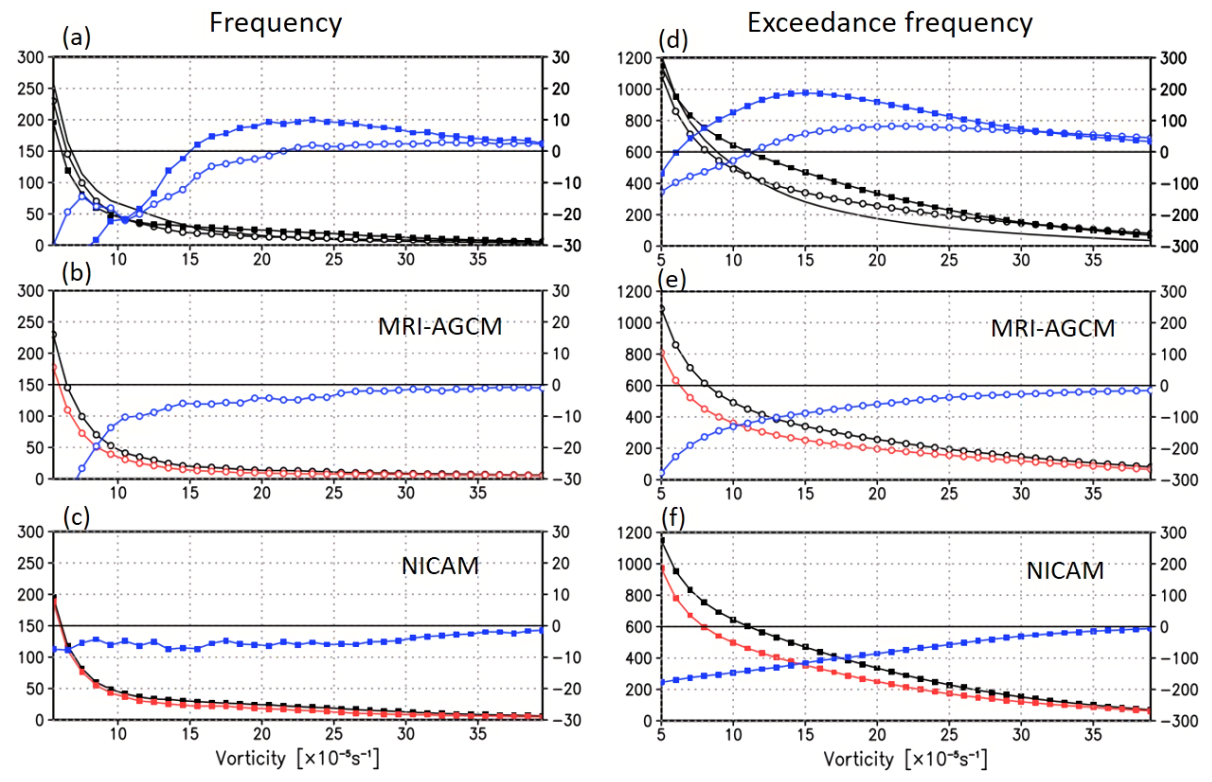

Fig. 2. Occurrence frequency distributions (unit is TC days per year) of vorticity (unit is $10^{-5} \mathrm{~s}^{-1}$ ) at $850 \mathrm{hPa}$. Frequency distributions (left panels) and exceedance frequency distributions (right panels). Thin black lines without line markers (in top panels) indicate JRA-55 (observation). For the lines with line markers, open circles indicate MRI-AGCM and closed squares indicate NICAM. Black (red) lines indicate present (future) climate simulations. Blue lines, with right hand side axes, indicate model bias (top panels), and future changes (middle and bottom panels). 

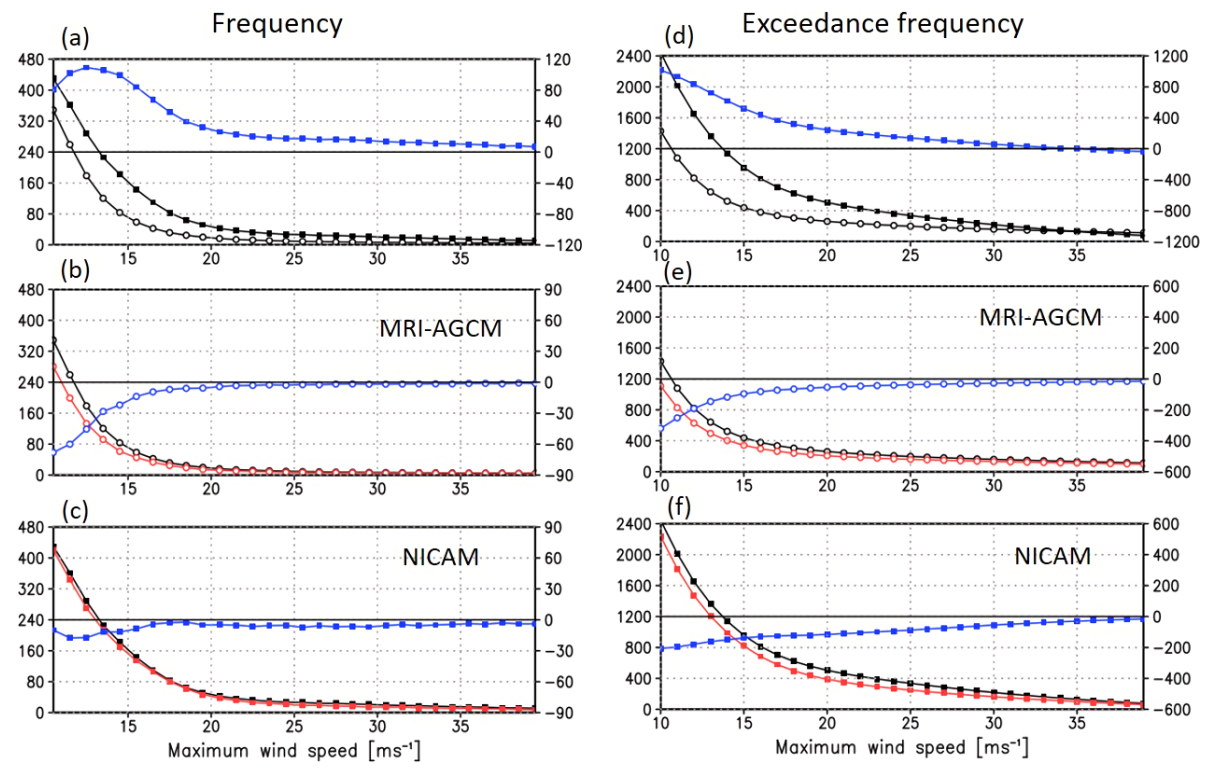

Fig. 3. Same as Fig. 2 but for the occurrence frequency distributions of maximum surface wind speed associated with the 850 hPa vortices. Range of the wind speed spans from $10 \mathrm{~m} \mathrm{~s}^{-1}$ to $40 \mathrm{~m} \mathrm{~s}^{-1}$ with $1 \mathrm{~m} \mathrm{~s}^{-1}$ bin size. Black (red) lines indicate present (future) climate simulations. Blue lines, with right hand side axes, indicate intermodal difference (NICAM-MRI-AGCM) (top panels), and future changes (middle and bottom panels).

Table 1. Occurence frequency (TC days per year) of each TC category. p-values are calculated using Mann-WhitneyWilcoxon test. Asterisk $\left(^{*}\right)$ indicates that the change is statistically significant $(p<0.05)$.

\begin{tabular}{|c|c|c|c|c|c|c|c|c|}
\hline & $\begin{array}{c}\text { TC Category } \\
\text { Max Wind Speed } \\
\left(\mathrm{m} \mathrm{s}^{-1}\right)\end{array}$ & $\begin{array}{c}\text { SEEDS } \\
10-17\end{array}$ & $\begin{array}{c}\mathrm{TS} \\
17-33\end{array}$ & $\begin{array}{l}\text { CAT1 } \\
33-43\end{array}$ & $\begin{array}{l}\text { CAT2 } \\
43-50\end{array}$ & $\begin{array}{l}\text { CAT3 } \\
50-59\end{array}$ & $\begin{array}{l}\text { CAT4 } \\
59-70\end{array}$ & $\begin{array}{c}\text { CAT5 } \\
70-\end{array}$ \\
\hline MRI-AGCM & $\begin{array}{l}\text { Present }(\mathrm{P}) \\
\text { Future }(\mathrm{F}) \\
\mathrm{F}-\mathrm{P} \\
(\mathrm{F}-\mathrm{P}) / \mathrm{P}(\%) \\
\text { p-value }\end{array}$ & $\begin{array}{c}1091.7 \\
844.4 \\
-247.3 \\
-22.7^{*} \\
<0.001\end{array}$ & $\begin{array}{l}194.8 \\
144.8 \\
-50.0 \\
-25.7^{*} \\
<0.001\end{array}$ & $\begin{aligned} & 46.0 \\
& 34.8 \\
- & -11.2 \\
- & 24.4^{*} \\
< & 0.001\end{aligned}$ & $\begin{array}{c}31.8 \\
24.8 \\
-6.9 \\
-21.8^{*} \\
<0.001\end{array}$ & $\begin{array}{r}37.8 \\
33.1 \\
-4.7 \\
-12.4 \\
0.091\end{array}$ & $\begin{array}{r}23.2 \\
21.0 \\
-2.2 \\
-9.3 \\
0.472\end{array}$ & $\begin{array}{c}3.1 \\
5.4 \\
2.4 \\
76.1^{*} \\
0.020\end{array}$ \\
\hline NICAM & $\begin{array}{l}\text { Present }(\mathrm{P}) \\
\text { Future }(\mathrm{F}) \\
\mathrm{F}-\mathrm{P} \\
(\mathrm{F}-\mathrm{P}) / \mathrm{P}(\%) \\
\mathrm{p}-\text {-value }\end{array}$ & $\begin{array}{c}1740.8 \\
1659.6 \\
-81.2 \\
-4.7^{*} \\
<0.001\end{array}$ & $\begin{array}{c}541.7 \\
455.4 \\
-86.3 \\
-15.9^{*} \\
<0.001\end{array}$ & $\begin{array}{c}122.4 \\
87.5 \\
-34.9 \\
-28.5^{*} \\
<0.001\end{array}$ & $\begin{array}{r}31.9 \\
26.6 \\
-5.3 \\
-16.6 \\
0.084\end{array}$ & $\begin{array}{r}7.2 \\
8.1 \\
0.9 \\
12.0 \\
0.542\end{array}$ & $\begin{array}{r}0.2 \\
0.4 \\
0.2 \\
72.7 \\
0.881\end{array}$ & $\begin{array}{l}0.0 \\
0.0 \\
0.0\end{array}$ \\
\hline
\end{tabular}
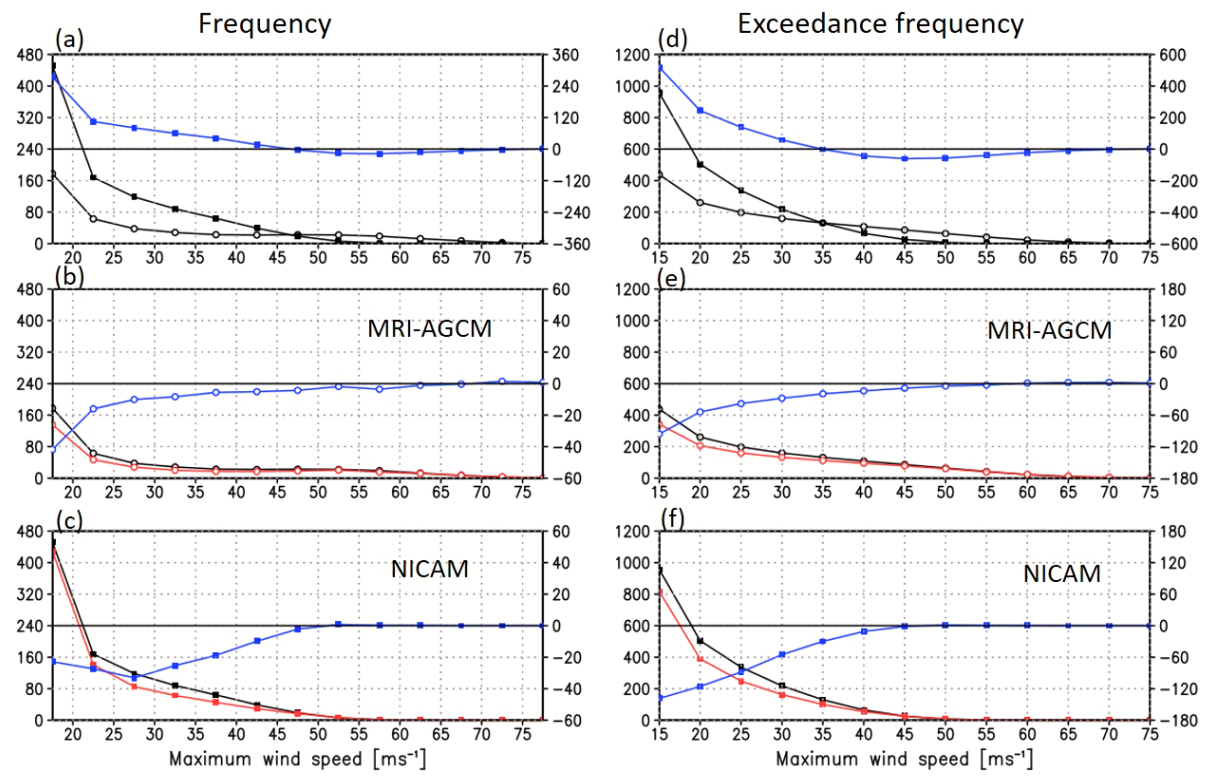

Fig. 4. Same as Fig. 3 but for TCs. Range of the wind speed spans from $15 \mathrm{~m} \mathrm{~s}^{-1}$ to $80 \mathrm{~m} \mathrm{~s}^{-1}$ with $5 \mathrm{~m} \mathrm{~s}^{-1}$ bin size. 
(a)
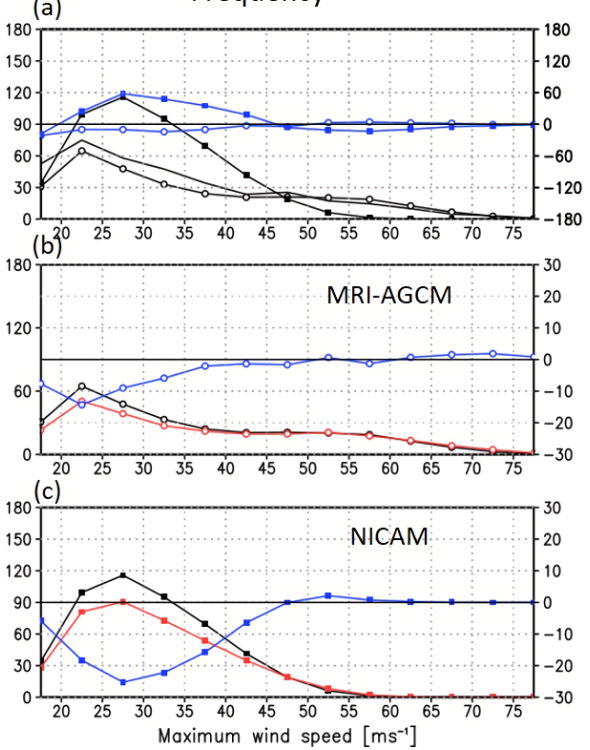

(d) Exceedance frequency
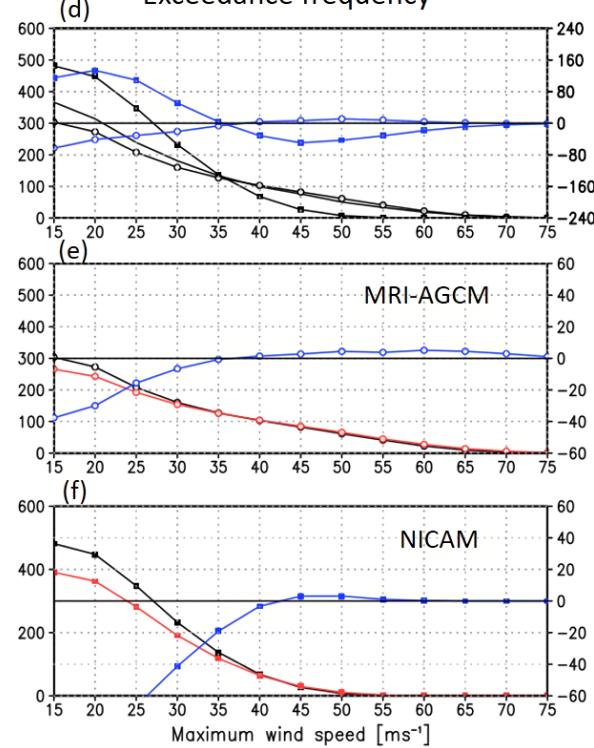

Fig. 5. Same as Fig. 4 but for the occurrence frequency calculated using wind speed in the TC tracking data. Thin black lines without line markers indicate Best Track (observation). Black (red) lines with line markers indicate present (future) climate simulations. Blue lines, with right hand side axes, indicate model bias (top panels), and future changes (middle and bottom panels).

is different, but the exceedance frequencies are the same for the same wind speed. As in Fig. 3, both models show a clear reduction of the occurrence frequency of the maximum wind speed in the future warmer climate. However, the difference is only marginal for the intense TCs with the surface maximum wind larger than $50 \mathrm{~m} \mathrm{~s}^{-1}$.

In order to compare the observed and simulated occurrence frequency distribution of the surface maximum wind speed of TCs, Fig. 5a shows the frequency distributions using the surface maximum wind in Best track Data and simulated TC tracking data. We applied the TC tracking scheme used in Yamada et al. (2017) to MRI-AGCM simulation data as well with a slight change. In the TC tracking scheme, the $850 \mathrm{hPa}$ vorticity threshold is 1.1 $\times 10^{-3} \mathrm{~s}^{-1}$ for NICAM but $2.0 \times 10^{-4}$ for lower resolution MRIAGCM, surface maximum wind speed threshold is $17.5 \mathrm{~m} \mathrm{~s}^{-1}$ and minimum duration time is 36 hour. In Fig. 5a we can see considerably large positive bias in the NICAM present climate simulation for the maximum surface wind speed between $20 \mathrm{~m} \mathrm{~s}^{-1}$ and $45 \mathrm{~m} \mathrm{~s}^{-1}$. We also note that there is a peak in the frequency distribution at $20-25 \mathrm{~m} \mathrm{~s}^{-1}$ for MRI-AGCM and Best Track Data, and at $25-30 \mathrm{~m} \mathrm{~s}^{-1}$ for NICAM, which is not seen in Fig. 4. The primary reason for this peak is that only the data with the wind speed larger than $17.5 \mathrm{~m} \mathrm{~s}^{-1}$ is included in the TC tracking data for the wind speed bin 15-20 m s . Another possible reason is that weak short lived TCs with duration time shorter than 36 hours are excluded in the TC tracking for the simulation data.

As in Fig. 4, Fig. 5 shows a clear reduction of the occurrence frequency of the maximum wind speed for weaker or medium strength TCs in the future warmer climate in the both models. However, the difference is only marginal for the intense TCs with the surface maximum wind larger than $50 \mathrm{~m} \mathrm{~s}^{-1}$.

Table 1 shows the occurrence frequency (TC days per year) of each TC category. We can see a clear (statistically significant) reduction of the frequency of TC seeds and weak TC categories (TS to CAT2). For the intense TC categories (CAT3-5), the changes are marginal in terms of the frequency. However, there are some very large increases in the frequency of the most intense TCs in terms of fractional changes, $73 \%$ for CAT4 in NICAM (statistically not significant) and $76 \%$ for CAT5 in MRI-AGCM (statistically significant).

\section{Discussion}

The main results of this study seem to be reasonable. The reduction of the occurrence frequency of TC seeds and weak TCs is consistent with the upward mass flux hypothesis (Sugi et al. 2012; Sugi et al. 2015), and the increase in the frequency of the most intense TCs is consistent with the MPI theory (Emanuel 1987).

In order to examine the possible future changes in TC activity, we used occurrence frequency analysis (OFA) in this study. The OFA method, which investigates the entire frequency distribution ranging from $\mathrm{TC}$ seeds to the most intense TCs, seems to have some advantages compared to the TC genesis frequency (TGF) analysis and lifetime maximum intensity (LMI) analysis. By using the OFA, we could clearly show a model bias or inter-model differences in the frequency distribution (Fig. 5). One advantage is that we have more data to estimate the frequency distributions. We have about 20 data on average for each TC ( 5 days of average lifetime of a TC and 4 six hourly data in a day), while we have only one data for TGF and LMI for each TC. Another advantage of OFA is that we can estimate occurrence frequency of TCs in a small region where the information of future changes in TC activity is needed for risk assessment. In case we do not have sufficient number of data in the region to estimate the regional occurrence frequency of extreme events such as intense TCs, some technique used in extreme value analysis (EVA) may be useful (Coles 2001; Sugi et al. 2017).

We have noted that in Fig. 5a there is a large bias in the occurrence frequency distribution even in the high resolution AGCMs. Particularly in the NICAM (14 km resolution) simulation, there is a very large positive bias for the TC maximum surface wind speed between $20 \mathrm{~m} \mathrm{~s}^{-1}$ and $45 \mathrm{~m} \mathrm{~s}^{-1}$, and the simulated frequency is very small for the wind speeds larger than $50 \mathrm{~m} \mathrm{~s}^{-1}$. There is no CAT5 TCs in NICAM simulation and CAT4 TCs may be interpreted as the most intense TCs in NICAM (Table 1). In order to quantitatively assess the future changes in the frequency of very intense TCs in such high resolution models, some bias correction (intensity adjustment or frequency adjustment) as applied to lower resolution models (Sugi et al. 2016; Yoshida et al. 2017), should be applied to these models. 


\section{Conclusions}

In relation to projections of tropical cyclone (TC) frequency in a future warmer climate, there is a debate on whether the global frequency of TC seeds (weak pre-storm vortices) will increase or not (Emanuel 2013; Vecchi et al. 2019). We examined changes in the frequency of TC seeds by occurrence frequency analysis (OFA) of vortex intensity (vorticity or maximum wind speed). We directly counted the number of vortices with various intensities in simulations for present and future climates with two high resolution global atmospheric models, $20 \mathrm{~km}$ resolution MRI-AGCM and $14 \mathrm{~km}$ resolution NICAM. By using the OFA we could show a clear reduction of the occurrence frequency of TC seeds $(850 \mathrm{hPa}$ vortices with surface maximum wind speed between $10 \mathrm{~m} \mathrm{~s}^{-1}$ and $17 \mathrm{~m} \mathrm{~s}^{-1}$ ) and relatively weak (CAT2 or weaker) TCs in a future warmer climate, with an increase in the occurrence frequency of the most intense (CAT5) TCs.

Advantages of the OFA were also discussed. By using the OFA, we can estimate future changes in occurrence frequency of TCs in a small region where the information of future changes in TC activity is needed for risk assessment. Also by using the OFA, we can clearly show a model bias or inter-model differences in the frequency distribution. Understanding the reason of these model bias and inter-model difference is an important subject of future work. The OFA seems a useful method to estimate the future changes in TC frequency distribution ranging from TC seeds to the most intense TCs.

\section{Acknowledgements}

This study was partly supported by the Ministry of Education, Culture, Sports, Science and Technology (MEXT) of Japan under the framework of the TOUGOU Program (JPMXD0717935561), and partly supported by FLAGSHIP2020 Project (hp190152) of MEXT.

Edited by: T.-Y. Koh

\section{Supplements}

Supplement Figure S1 shows the one month animation of Figure 1.

\section{References}

Bhatia, K., G. Vecchi, H. Murakami, S. Underwood, and J. Kossin, 2018: Projected response of tropical cyclone intensity and intensification in a global climate model. J. Climate, 31, 8281-8303, doi:10.1175/JCLI-D-17-0898.1.

Coles, S., 2001: An Introduction to Statistical Modeling of Extreme Values. Springer Verlag, London, UK.

Emanuel, K., 1987: The dependency of hurricane intensity on climate. Nature, 326, 483-485.

Emanuel, K., 2013: Downscaling CMIP5 climate models shows increased tropical cyclone activity over the 21 st century. Proc. Nat. Acad. Sci., 110, 12219-12224.

Knapp, K. R., M. C. Kruk, D. H. Levinson, H. J. Diamond, and C. J. Neumann, 2010: The International Best Track Archive for Climate Stewardship (IBTrACS): Unifying tropical cyclone best track data. Bull. Amer. Meteor. Soc., 91, 363-376.

Knutson, T., and co-authors, 2020: Tropical cyclone and climate change assessment Part II. Projected responses to anthropogenic waring. Bull. Amer. Meteor. Soc., doi:10.1175/BAMSD-18-0194.1.

Kobayashi, S., and co-authors, 2015: The JRA-55 reanalysis: General specifications and basic characteristics. J. Meteor. Soc. Japan, 93, 5-48, doi:10.2151/jmsj.2015-001.

Kodama, C., and co-authors, 2015: A 20-year climatology of a NICAM AMIP-type simulation. J. Meteor. Soc. Japan, 93, 393-424, doi:10.2151/jmsj.2015-024.

Meehl, G., C. Covey, T. Delworth, M. Latif, B. McAvaney, J. Mitchell, R. Stouffer, and K. Taylor, 2007: The WCRP CMIP3 multimodel dataset: A new era in climate change research. Bull. Amer. Meteor. Soc., 88, 1383-1394, doi: 10.1175/BAMS-88-9-1383.

Mizuta, R., and co-authors, 2012: Climate simulations using MRIAGCM3.2 with 20-km grid. J. Meteor. Soc. Japan, 90A, 233-258, doi:10.2151/jmsj.2012-A12.

Satoh, M., T. Matsuno, H. Tomita, H. Miura, T. Nasuno, and S. Iga, 2008: Nonhydrostatic Icosahedral Atmospheric Model (NICAM) for global cloud resolving simulations. J. Comp. Phys., 227, 3486-3514, doi:10.1016/j.jcp.2007.02.006.

Satoh, M., H. Tomita, H. Yashiro, and co-authors, 2014: The nonhydrostatic icosahedral atmospheric model: Description and development. Prog. in Earth and Planet. Sci. 1, 18, doi: 10.1186/s40645-014-0018-1.

Satoh, M., Y. Yamada, M. Sugi, C. Kodama, and A. T. Noda, 2015: Constraint on future change in global frequency of tropical cyclones due to global warming. J. Meteor. Soc. Japan, 93, 489-500, doi:10.2151/jmsj.2015-025.

Sugi, M., and J. Yoshimura, 2012: Decreasing trend of tropical cyclone frequency in 228-year high-resolution AGCM simulations. Geophys. Res. Lett., 39, L19805, doi:10.1029/2012 GL053360.

Sugi, M., H. Murakami, and J. Yoshimura, 2012: On the mechanism of tropical cyclone frequency changes due to global warming. J. Meteor. Soc. Japan, 90A, 397-408.

Sugi, M., K. Yoshida, and H. Murakami, 2015: More tropical cyclones in a cooler climate? Geophys. Res. Lett., 42, 67806784, doi:10.1002/2015GL064929.

Sugi, M., H. Murakami, and K. Yoshida, 2016: Projection of future changes in the frequency of intense tropical cyclones. Climate Dyn., 49, 619-632, doi:10.1007/s00382-016-3361-7.

Sugi, M., Y. Imada, T. Nakaegawa, and K. Kamiguchi, 2017: Estimating probability of extreme rainfall over Japan using extended regional frequency analysis. Hydro. Res. Lett., 11, 19-23, doi:10.3178/hrl.11.19.

Taylor, K. E., R. J. Stouffer, and G. A. Meehl, 2012: An overview of CMIP5 and the experiment design. Bull. Amer. Meteor. Soc., 93, 485-498.

Vecchi, and co-authors, 2019: Tropical cyclone sensitivities to CO2 doubling: Roles of atmospheric resolution, synoptic variability and background climate changes. Climate Dyn., doi:10.1007/s00382-019-04913-y.

Yamada, Y., M. Satoh, M. Sugi, C. Kodama, A. T. Noda, M. Nakano, and T. Nasuno, 2017: Response of tropical cyclone activity and structure to global warming in a high-resolution global nonhydrostatic model. J. Climate, 30, 9703-9724, doi:19.1175/JCLI-D-0068.1.

Yoshida, K., M. Sugi, R. Mizuta, H. Murakami, and M. Ishii, 2017: Future changes in tropical cyclone activity in high resolution large-ensemble simulations. Geophys. Res. Lett., doi:10.1002/2017GL075058.

Manuscript received 27 December 2019, accepted 11 March 2020 SOLA: https://www.jstage.jst.go.jp/browse/solal 